\title{
Callosal Disconnection Syndrome Associated with Relapsing Polychondritis
}

\author{
Toru Baba ${ }^{1}$, Shigenori Kanno ${ }^{1-3}$, Tomomi Shijo ${ }^{2,3}$, Yoshiyuki Nishio ${ }^{1}$, Osamu Iizuka ${ }^{1}$, \\ Naoto Kamimura ${ }^{4}$, Tomonori Ishii ${ }^{5}$ and Etsuro Mori ${ }^{1}$
}

\begin{abstract}
Relapsing polychondritis (RP) is a rare inflammatory disorder of the cartilagenous structures, and it sometimes involves the central nervous system. Encephalitis associated with RP causes a wide variety of symptoms according to the affected sites. We herein report the first case of 72-year-old right-handed man who developed acute meningoencephalitis associated with RP involving the corpus callous. After immunosuppressive therapy, his symptoms dramatically improved, but difficulty in performing bimanual movements with occasional diagonistic dyspraxia in his right hand remained. Because callosal signs are easily missed, especially in acute settings, it would be useful to know that RP can sometimes cause callosal disconnection syndrome.
\end{abstract}

Key words: relapsing polychondritis, callosal dysconnection syndrome, diagonistic dyspraxia

(Intern Med 55: 1191-1193, 2016)

(DOI: 10.2169/internalmedicine.55.5345)

\section{Introduction}

Relapsing polychondritis (RP) is a rare relapsing and remitting inflammatory disorder of the cartilagenous structures (1). RP has been reported to cause central nervous system vasculitis in rare cases, thus leading to various neurological symptoms, such as meningitis, encephalitis (2), parkinsonism (3) and dementia (4), according to the site of lesions. We herein describe, for the first time, that RP may sometimes cause callosal disconnection syndrome.

\section{Case Report}

A 72-year old right-handed man with 16 years of education was found lying drowsy on the floor and thereafter was brought to the nearest hospital by ambulance. His past medical history included diabetes mellitus, hypertension, postoperative thoracic aortic aneurysm and cured gastric MALT (mucosa-associated lymphatic tissue) lymphoma. Two years before this presentation, at 70 years of age, he presented with eruptions over his body and extremities. He was given $60 \mathrm{mg} /$ day oral prednisolone and the eruptions thereafter disappeared, however he developed pain and swelling in his left ear while tapering prednisolone to $5 \mathrm{mg} /$ day. A biopsy of the auricular cartilage showed perichondral inflammation with lymphocytic infiltration. A diagnosis of RP was thus made and oral prednisolone was increased to $10 \mathrm{mg} / \mathrm{day}$ with a complete recovery of the symptoms. He then remained in good health until 72 years of age, when he developed a high fever and drowsiness two days before admission.

On admission, the patient was febrile and somnolent. Dysarthria and neck stiffness were evident. Blood tests revealed an elevated white blood cell count and C-reactive protein concentration. A cerebrospinal fluid (CSF) examination showed pleocytosis of $781 / \mathrm{mm}^{3}$ white blood cells with $87 \%$ polymoropholeucocytes, a glucose level of $38 \mathrm{mg} / \mathrm{dL}$ (blood glucose $149 \mathrm{mg} / \mathrm{dL}$ ), and a total protein of $582 \mathrm{mg} /$ dL. A computed tomography scan of the brain showed no obvious abnormalities. With the presumptive diagnosis of acute bacterial meningitis, he was treated with dexametha-

\footnotetext{
${ }^{1}$ Department of Behavioral Neurology \& Cognitive Neuroscience, Tohoku University Graduate School of Medicine, Japan, ${ }^{2}$ Department of Neurology, South Miyagi Medical Center, Japan, ${ }^{3}$ Department of Neurology, Tohoku University Graduate School of Medicine, Japan, ${ }^{4}$ Department of Neuropsychiatry, Kochi Medical School, Japan and ${ }^{5}$ Deartment of Hematology and Rheumatology, Tohoku University Graduate School of Medicine, Japan

Received for publication March 13, 2015; Accepted for publication August 6, 2015

Correspondence to Dr. Toru Baba, t-baba@med.tohoku.ac.jp
} 
a.

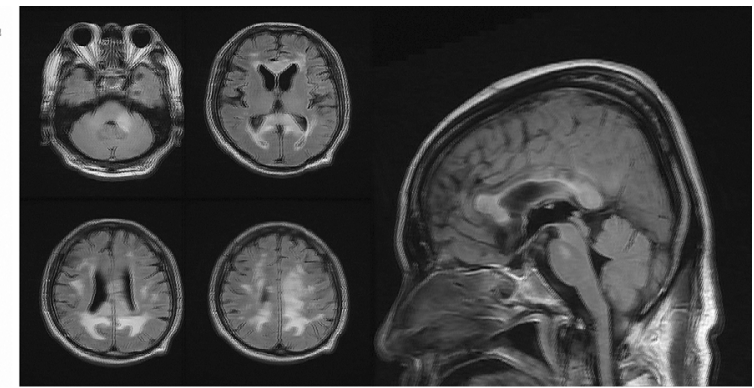

b.

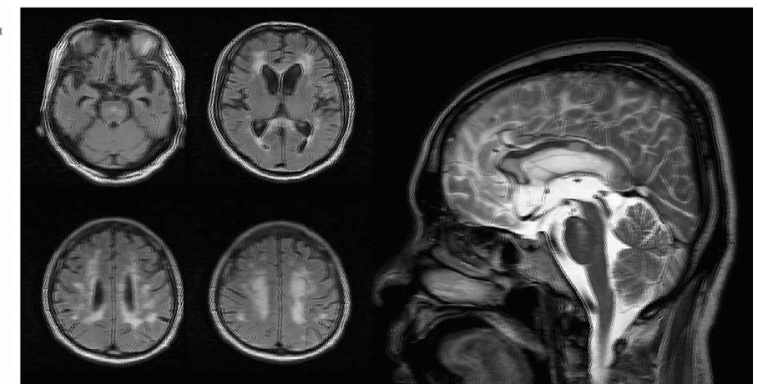

Figure 1. Axial and sagittal fluid attenuation inversion recovery (FLAIR) and T2-weighted sequences in brain MRI. a: At presentation: lesions are observed in the corpus callosum and in the left inferior cerebellar peduncle in addition to diffuse subcortical white matter abnormalities. b: After 4 months of treatment: the subcortical white matter hyperintense lesions became less remarkable, but the lesions in the genu and splenium of the corpus callosum persisted.

sone $32 \mathrm{mg} /$ day for 4 days and antibiotics. Three days later, the patient demonstrated a dramatic improvement of his CSF abnormalities. Brain magnetic resonance imaging (MRI) showed diffuse subcortical white matter abnormalities without contrast-enhancement including the corpus callosum (Fig. 1a). There was no involvement of the medial frontal cortex. All CSF specimens were negative for bacteria, virus, and fungi or abnormal cells. Acute bacterial meningitis was thought to be unlikely, and alternatively meningoencephalitis associated with RP was highly suspected. All antibiotics were discontinued and oral prednisolone at a dose of $60 \mathrm{mg}$ / day was started, and thereafter his condition improved gradually.

One month after admission, he was transferred to our hospital and oral prednisolone was gradually tapered to $10 \mathrm{mg}$ / day and pulsed intravenous cyclophosphamide $(500 \mathrm{mg}$ monthly for 4 months) was administered. At that time, he complained of speech difficulty and sometimes reported that his left hand would not respond as intended. The results of a neurological examination were not remarkable, except for the observed neurocognitive deficits. He was alert but disoriented as to the time and place, and he achieved a score of 13 on the Mini-Mental State Examination (MMSE). His speech was slow and often stopped due to occasional stuttering, but his articulation and prosody were adequate. Circumlocutions and semantic paraphasias were sometimes noted in his spontaneous speech. The abilities of comprehension, repetition, and reading were preserved, but he had difficulty in writing especially with his left hand (Fig. 2a, b). He could not perform pentagon copy, cube copy and clock drawing in either hand, thus indicating a visuospatial impairment (Fig. 2e). Furthermore, mild left-sided hemispatial neglect was observed when copying line drawings with his right-hand (Fig. 2c, d). Ideomotor apraxia of both hands was observed on verbal command and on imitation. He could not identify his left hand fingers or objects placed in his left hand with his eyes closed, but he was able to do so under visual observation.

After a rehabilitation period of 4 months in our hospital, his cognitive status improved significantly and his MMSE score improved to 21 . His speech became faster and more fluent except for some stuttering. He became able to write with his right hand proficiently. Visuospatial impairment also improved remarkably, but he could not copy a doublepentagon or perform clock drawing (Fig. 2f). Apraxia of both hands was markedly diminished. Despite the significant improvement, he still had difficulty in performing bimanual movements, such as tying a string, lacing up shoes and fastening a button, as the right hand would not help the left and at times even tried to interfere with the action of the left hand (diagonistic dyspraxia), even though he often felt a loss of control of his left hand. On repeated MRI, subcortical white matter lesions became less remarkable and the lesions in the corpus callosum shrunk (Fig. 1b).

\section{Discussion}

Corpus callosum involvement associated with RP has been inferred on MRIs in a previous report, although callosal disconnection syndrome has never been previously described (5). Because callosal signs and symptoms are prone to be missed, especially in acute settings (6) which can lead to a misestimation of the disease severity, it would therefore be useful to know that RP can sometimes cause callosal disconnection syndrome accompanied by encephalitis.

It is noteworthy that the diagonistic dyspraxia in the present case was in the right hand, although it generally occurs in the left (non-dominant) hand in right-handed individuals $(7,8)$. This reversed lateralization can be explained by the possibility that our patient had a mixed cortical dominance. In general, dysgraphia and ideomotor apraxia emerge in one's non-dominant hand in callosal disconnection syndrome $(9,10)$, but these were observed in both hands at the initial stage, which also suggests an altered cortical lateralization in this patient. Nevertheless, tactile anomia (or astereognosia) of the left hand suggests that the left hemisphere dominancy of language function (10).

Another peculiar thing to be noted in this case is a discrepancy between the affected-sides of diagonistic dyspraxia and the loss of the sense of agency. It is possible that the left hemisphere accounts for the verbal expression of a feeling of the loss of control over his left hand, even if the left hand actually manifested the patient's intention.

In the current study, we describe a patient who presented with right-sided diagonistic dyspraxia due to callosal discon- 


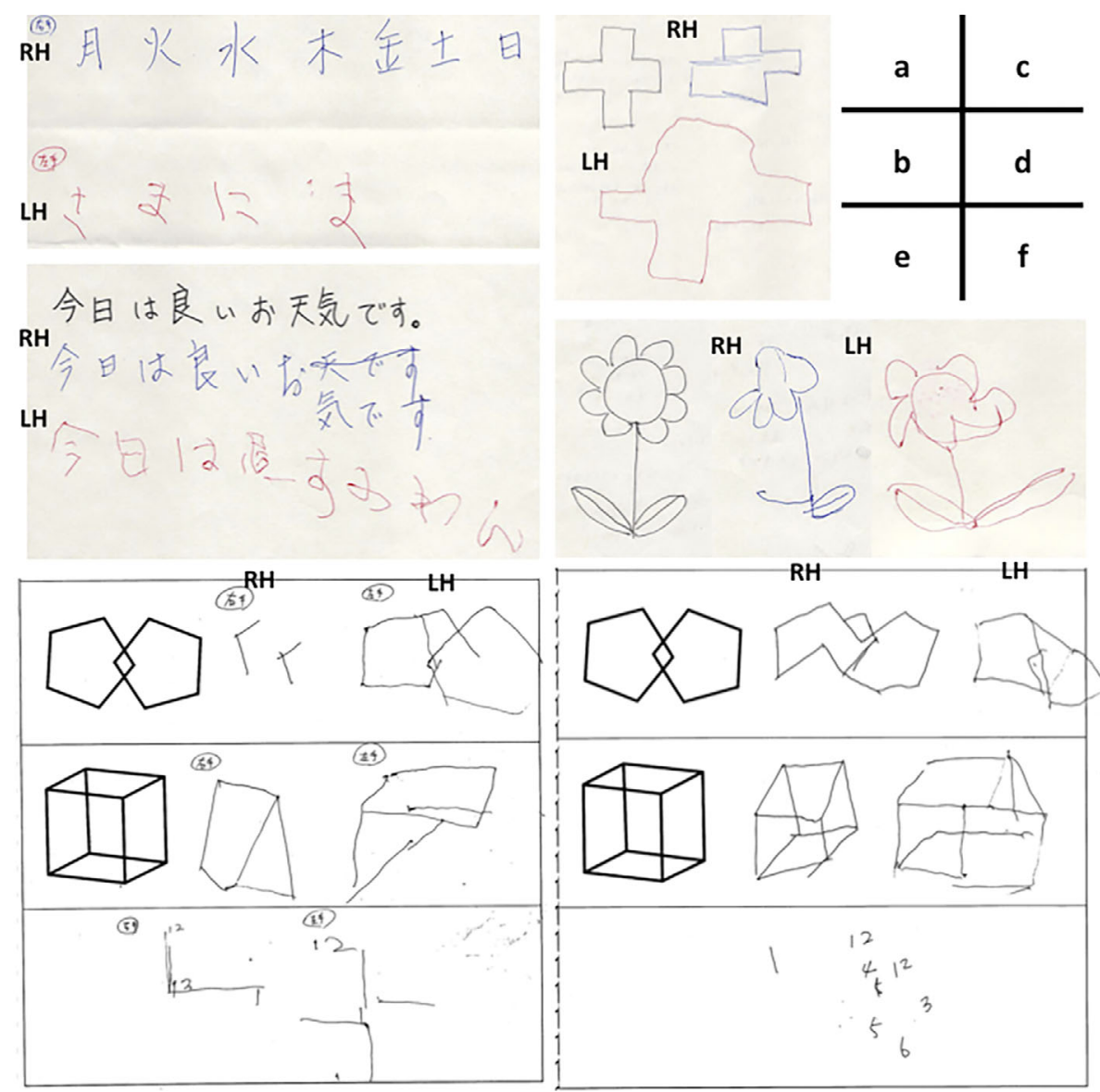

Figure 2. Samples of patient's writing, figure copying and clock drawing. a: When instructed to recite the days of the week, the patient made only a minor error with his right-hand, but he could only write meaningless characters with his left-hand. b: When asked to copy a simple sentence, the patient made only a minor mistake with the right-hand, but he could write only a nonsensical phrase with the left-hand. $c$ and d: Mild left-sided hemispatial neglect was observed when copying line drawings with his right-hand. e: He could not copy line drawings or draw a clock at presentation. f: After 4 months of treatment, he became able to copy a cube with his right hand, but he remained unable to copy a double-pentagon or draw a clock.

nection secondary to RP. The present report adds an additional case of callosal disconnection syndrome in a RP patient. RP should therefore be considered as a rare cause of alien hand syndrome.

The authors state that they have no Conflict of Interest (COI).

\section{Acknowledgement}

We thank Kanae Izumi for performing cognitive tests and rehabilitation for the patient.

\section{References}

1. McAdam LP, O'Hanlan MA, Bluestone R, Pearson CM. Relapsing polychondritis: prospective study of 23 patients and a review of the literature. Medicine 55: 193-215, 1976.

2. Sundaram MB, Rajput AH. Nervous system complications of relapsing polychondritis. Neurology 33: 513-515, 1983.

3. Defer GL, Danaila T, Constans JM, Derache N. Relapsing poly- chondritis revealed by basal ganglia lesions. Mov Disord 27: 1094-1096, 2012.

4. Head E, Starr A, Kim RC, et al. Relapsing polychondritis with features of dementia with Lewy bodies. Acta Neuropathol 112: 217-225, 2006.

5. Garcia-Egido A, Gutierrez C, De la Fuente C, Gomez F. Relapsing polychondritis-associated meningitis and encephalitis: response to infliximab. Rheumatology (Oxford) 50: 1721-1723, 2011.

6. Berlucchi G. Frontal callosal disconnection syndromes. Cortex 48 : 36-45, 2012.

7. Feinberg TE, Schindler RJ, Flanagan NG, Haber LD. Two alien hand syndromes. Neurology 42: 19-24, 1992.

8. Tanaka Y, Yoshida A, Kawahata N, Hashimoto R, Obayashi T. Diagonistic dyspraxia. Clinical characteristics, responsible lesion and possible underlying mechanism. Brain 119: 859-873, 1996.

9. McKeever WF, Sullivan KF, Ferguson SM, Rayport M. Typical cerebral hemisphere disconnection deficits following corpus callosum section despite sparing of the anterior commissure. Neuropsychologia 19: 745-755, 1981 .

10. Gazzaniga MS. Cerebral specialization and interhemispheric communication: does the corpus callosum enable the human condition? Brain 123: 1293-1326, 2000.

(C) 2016 The Japanese Society of Internal Medicine http://www.naika.or.jp/imonline/index.html 